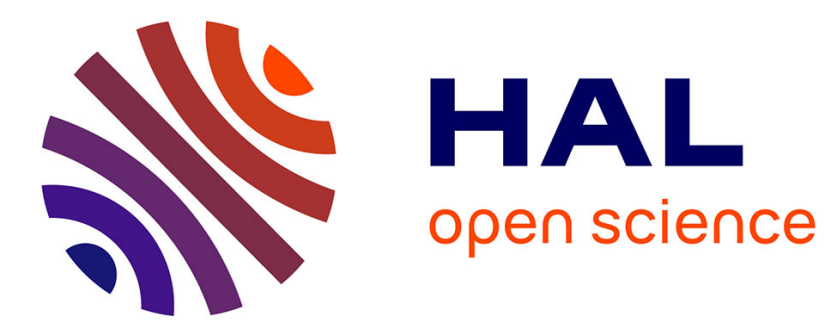

\title{
k-tuple chromatic number of the cartesian product of graphs
}

Flavia Bonomo, Ivo Koch, Pablo Torres, Mario Valencia-Pabon

\section{To cite this version:}

Flavia Bonomo, Ivo Koch, Pablo Torres, Mario Valencia-Pabon. k-tuple chromatic number of the cartesian product of graphs. 2014. hal-01103534

\section{HAL Id: hal-01103534 \\ https://hal.science/hal-01103534}

Preprint submitted on 14 Jan 2015

HAL is a multi-disciplinary open access archive for the deposit and dissemination of scientific research documents, whether they are published or not. The documents may come from teaching and research institutions in France or abroad, or from public or private research centers.
L'archive ouverte pluridisciplinaire HAL, est destinée au dépôt et à la diffusion de documents scientifiques de niveau recherche, publiés ou non, émanant des établissements d'enseignement et de recherche français ou étrangers, des laboratoires publics ou privés. 


\title{
$k$-tuple chromatic number of the cartesian product of graphs *
}

\author{
Flavia Bonomo $^{\dagger} \quad$ Ivo Koch ${ }^{\ddagger}$ Pablo Torres ${ }^{\S} \quad$ Mario Valencia-Pabon $₫$
}

\begin{abstract}
A $k$-tuple coloring of a graph $G$ assigns a set of $k$ colors to each vertex of $G$ such that if two vertices are adjacent, the corresponding sets of colors are disjoint. The $k$-tuple chromatic number of $G, \chi_{k}(G)$, is the smallest $t$ so that there is a $k$-tuple coloring of $G$ using $t$ colors. It is well known that $\chi(G \square H)=\max \{\chi(G), \chi(H)\}$. In this paper, we show that there exist graphs $G$ and $H$ such that $\chi_{k}(G \square H)>\max \left\{\chi_{k}(G), \chi_{k}(H)\right\}$ for $k \geq 2$. Moreover, we also show that there exist graph families such that, for any $k \geq 1$, the $k$-tuple chromatic number of their cartesian product is equal to the maximum $k$-tuple chromatic number of its factors.
\end{abstract}

Keywords: $k$-tuple colorings, Cartesian product of graphs, Kneser graphs, Cayley graphs, Hom-idempotent graphs.

\section{Introduction}

A classic coloring of a graph $G$ is an assignment of colors (or natural numbers) to the vertices of $G$ such that any two adjacent vertices are assigned different colors. The smallest number $t$ such that $G$ admits a coloring with $t$ colors (a $t$-coloring) is called the chromatic number of $G$ and is denoted by $\chi(G)$. Several generalizations of the coloring problem have been introduced in the literature, in particular, cases in which each vertex is assigned not only a color but a set of colors, under different restrictions. One of these variations is the $k$-tuple coloring introduced independently by Stahl [11] and Bollobás and Thomason [3]. A $k$-tuple

*Partially supported by MathAmSud Project 13MATH-07 (Argentina-Brazil-Chile-France), UBACyT Grant 20020130100808BA, CONICET PIP 112-200901-00178 and 112-201201-00450CO and ANPCyT PICT 2012-1324 (Argentina)

${ }^{\dagger}$ CONICET and Dep. de Computación, FCEN, Universidad de Buenos Aires, Argentina. e-mail: fbonomo@dc.uba.ar

${ }^{\ddagger}$ Instituto de Ciencias, Universidad Nacional de General Sarmiento, Argentina. e-mail: ivo.koch@gmail.com

$\S$ Universidad Nacional de Rosario and CONICET, Rosario, Argentina. e-mail: ptorres@fceia.unr.edu.ar

IUniversité Paris-13, Sorbonne Paris Cité LIPN, CNRS UMR7030, Villetaneuse, France. Currently in Délégation at the INRIA Nancy - Grand Est. e-mail: valencia@lipn.univ-paris13.fr 
coloring of a graph $G$ is an assignment of $k$ colors to each vertex in such a way that adjacent vertices are assigned distinct colors. The $k$-tuple coloring problem consists into finding the minimum number of colors in a $k$-tuple coloring of a graph $G$, which we denote by $\chi_{k}(G)$. The cartesian product $G \square H$ of two graphs $G$ and $H$ has vertex set $V(G) \times V(H)$, two vertices being joined by an edge whenever they have one coordinate equal and the other adjacent. This product is commutative and associative up to isomorphism. There is a simple formula expressing the chromatic number of a cartesian product in terms of its factors:

$$
\chi(G \square H)=\max \{\chi(G), \chi(H)\} .
$$

The identity (1) admits a simple proof first given by Sabidussi [10]. The Kneser graph $K(m, n)$ has as vertices all $n$-element subsets of the set $[m]=\{1, \ldots, m\}$ and an edge between two subsets if and only if they are disjoint. We will assume in the rest of this work that $m \geq 2 n$, otherwise $K(m, n)$ has no edges. The Kneser graph $K(5,2)$ is the well known Petersen Graph. Lovász [9] showed that $\chi(K(m, n))=m-2 n+2$. The value of the $k$-tuple chromatic number of the Kneser graph is the subject of an almost 40-year-old conjecture of Stahl [11] which asserts that: if $k=q n-r$ where $q \geq 0$ and $0 \leq r<n$, then $\chi_{k}(K(m, n))=q m-2 r$. Stahl's conjecture has been confirmed for some values of $k, n$ and $m[11,12]$.

An homomorphism from a graph $G$ into a graph $H$, denoted by $G \rightarrow H$, is an edgepreserving map from $V(G)$ to $V(H)$. It is well known that an ordinary graph coloring of a graph $G$ with $m$ colors is an homomorphism from $G$ into the complete graph $K_{m}$. Similarly, an $n$-tuple coloring of a graph $G$ with $m$ colors is an homomorphism from $G$ into the Kneser graph $K(m, n)$.

A graph $G$ is said hom-idempotent if there is a homomorphism from $G \square G \rightarrow G$. We denote by $G \nrightarrow H$ if there exists no homomorphism from $G$ to $H$.

The clique number of a graph $G$, denoted by $\omega(G)$, is the maximum size of a clique in $G$ (i.e., a complete subgraph of $G$ ). Clearly, for any graphs $G$ and $H$, we have that $\chi(G) \geq \omega(G)$ (and so, $\left.\chi_{k}(G) \geq \chi_{k}\left(K_{\omega(G)}\right)=k \omega(G)\right)$ and, if there is an homomorphism from $G$ to $H$ then, $\chi(G) \leq \chi(H)$ (and so, $\left.\chi_{k}(G) \leq \chi_{k}(H)\right)$.

A stable set $S \subseteq V$ is a subset of pairwise non adjacent vertices of $G$. The stability number of $G$, denoted by $\alpha(G)$, is the largest cardinality of a stable set in $G$. Let $m \geq 2 n$. An element $i \in[m]$ is called a centre of a stable set $S$ of the Kneser graph $K(m, n)$ if it lies in each $n$-set in $S$.

Lemma 1 (Erdös-Ko-Rado [4]). If $m>2 n$, then $\alpha(K(m, n))=\left(\begin{array}{l}m-1 \\ n-1\end{array}\right)$. An independent set of $K(m, n)$ with size $\left(\begin{array}{c}m-1 \\ n-1\end{array}\right)$ has a centre $i$, for some $i \in[m]$.

Lemma 2 (Hilton-Milner [7]). If $m \geq 2 n$, then the maximum size of an stable set in $K(m, n)$ with no centre is equal to $1+\left(\begin{array}{c}m-1 \\ n-1\end{array}\right)-\left(\begin{array}{c}m-n-1 \\ n-1\end{array}\right)$.

A graph $G=(V, E)$ is vertex transitive if its automorphism group acts transitively on $V$, that is, for any pair of distinct vertices of $G$ there is an automorphism mapping one to the other one. It is well known that Kneser graphs are vertex transitive graphs [5]. 
Lemma 3 (No-Homomorphism Lemma, Albertson-Collins [1]). Let $G, H$ be graphs such that $H$ is vertex transitive and $G \rightarrow H$. Then, $\alpha(G) /|V(G)| \geq \alpha(H) /|V(H)|$.

In this paper, we show that equality (1) does not hold in general for $k$-tuple colorings of graphs. In fact, we show that for some values of $k \geq 2$, there are Kneser graphs $K(m, n)$ for which $\chi_{k}(K(m, n) \square K(m, n))>\chi_{k}(K(m, n))$. Moreover, we also show that there are families of graphs for which equality (1) holds for $k$-tuple colorings of graphs for any $k \geq 1$. As far as we know, our results are the first ones concerning the $k$-tuple chromatic number of cartesian product of graphs.

\section{Cartesian products of Kneser graphs}

Lemma 4. Let $G$ be a graph and let $k>0$. Then, $\chi_{k}(G \square G) \leq k \chi(G)$.

Proof. Clearly, $\chi_{k}(G \square G) \leq k \chi(G \square G)$. However, by equality (1) we know that $\chi(G \square G)=$ $\chi(G)$, and thus the lemma holds.

Corollary 1. $\chi_{k}(K(m, n) \square K(m, n)) \leq k \chi(K(m, n))=k(m-2 n+2)$.

Larose et al. [8] showed that no connected Kneser graph $K(m, n)$ is hom-idempotent, that is, for any $m>2 n$, there is no homomorphism from $K(m, n) \square K(m, n)$ to $K(m, n)$.

Lemma 5 ([8]). Let $m>2 n$. Then, $K(m, n) \square K(m, n) \not \rightarrow K(m, n)$.

Concerning the $k$-tuple chromatic number of some Kneser graphs, Stahl [11] showed the following results.

Lemma 6 ([11]). If $1 \leq k \leq n$, then $\chi_{k}(K(m, n))=m-2(n-k)$.

Lemma $7([11]) \cdot \chi_{k}(K(2 n+1, n))=2 k+1+\left\lfloor\frac{k-1}{n}\right\rfloor$, for $k>0$.

Lemma $8([11]) \cdot \chi_{r n}(K(m, n))=r m$, for $r>0$ and $m \geq 2 n$.

By using Lemma 8 we have the following result.

Lemma 9. Let $m>2 n$. Then, $\chi_{n}(K(m, n) \square K(m, n))>\chi_{n}(K(m, n))$.

Proof. By Lemma 8 when $r=1$, we have that $\chi_{n}(K(m, n))=m$. If $\chi_{n}(K(m, n) \square K(m, n))=m$, then there exists an homomorphism from the graph $K(m, n) \square K(m, n)$ to $K(m, n)$ which contradicts Lemma 5.

By Lemma 6, Lemma 9 and by using Corollary 1, we have that,

Corollary 2. Let $n \geq 2$. Then, $2 n+2 \leq \chi_{n}(K(2 n+1, n) \square K(2 n+1, n)) \leq 3 n$. In particular, when $n=2$, we have that $\chi_{2}(K(5,2) \square K(5,2))=6$.

In the case $k=2$ we have by Lemma 9, Lemma 6 and by Corollary 1, the following result. 
Corollary 3. Let $q>0$. Then, $q+4 \leq \chi_{2}(K(2 n+q, n) \square K(2 n+q, n)) \leq 2 q+4$.

By Corollary 3, notice that in the case when $k=n=2$ and $q \geq 1$, we must have that $\chi_{2}(K(q+4,2) \square K(q+4,2))>q+4$, otherwise there is a contradiction with Lemma 5 . This provides a gap of one unity between the 2-tuple chromatic number of the graph $K(q+4,2) \square K(q+4,2)$ and the graph $K(q+4,2)$. In the next Lemma 11, we will show that such a gap can be as large as desired. However, first we need to introduce the following.

It is well known that the chromatic index of a complete graph $K_{2 n}$ (i.e. the minimum number of colors needed to color the edges of $K_{2 n}$ such that any two incident edges be assigned different colors) on $2 n$ vertices is equal to $2 n-1$ (see [2]), where each color class $i$ (i.e. the subset of pairwise non incident edges colored with color $i$ ) has size $n$. Therefore, using this fact, we obtain the following result.

Lemma 10. Let $q \geq 1$. Then, the set of vertices of the Kneser graph $K(2 q+4,2)$ can be partitioned into $2 q+3$ disjoint cliques, each one with size $q+2$.

Proof. Notice that there is a natural bijection between the vertex set of $K(2 q+4,2)$ and the edge set of the complete graph $K_{2 q+4}$ with vertex set $[2 q+4]$ : each vertex $\{i, j\}$ in $K(2 q+4,2)$ is mapped to the edge $\{i, j\}$ in $K_{2 q+4}$. Now, there is a $(2 q+3)$-edge coloring of $K_{2 q+4}$ where each class color is a set of pairwise non incident edges with size $q+2$. Notice that two edges $e, e^{\prime} \in K_{2 q+4}$ are non incident edges if and only if $e \cap e^{\prime}=\emptyset$. Therefore, a class color of the edge-coloring of $K_{2 q+4}$ represents a clique of $K(m, n)$.

Lemma 11. Let $q>0$. Then, $\chi_{2}(K(2 q+4,2) \square K(2 q+4,2)) \geq 2 q+\left\lceil\frac{2}{3} q\right\rceil+5$.

Proof. First, recall that a stable set $X$ in $K(2 q+4,2)$ has size at most $2 q+3$ if $X$ has centre (see Lemma 1) and $|X| \leq 1+(2 q+4-1)-(2 q+4-2-1)=3$ if $X$ has no centre (see Lemma 2). Besides, by Lemma 10, observe that the vertex set of $K(2 q+4,2)$ can be partitioned in $2 q+3$ sets $S_{1}, \ldots, S_{2 q+3}$ such that each $S_{i}$ induces a $K_{q+2}$ for $i=1, \ldots, 2 q+3$. Consider the subgraph $H_{i}$ of $K(2 q+4,2) \square K(2 q+4,2)$ induced by $S_{i} \times V(K(2 q+4,2))$ for $i=1, \ldots, 2 q+3$. Let $I$ be a stable set in $K(2 q+4,2)$ and $I_{i}=I \cap H_{i}$ for $i=1, \ldots, 2 q+3$. Then $I_{i}^{v}=I_{i} \cap(\{v\} \times V(K(2 q+4,2)))$ is a stable set in $K(2 q+4,2)$ for each $v \in S_{i}$ and $i=1, \ldots, 2 q+3$.

Now, assume w.l.o.g that $r(r \leq q+2)$ stable sets $I_{i}^{1}, \ldots, I_{i}^{r}$ have distinct centre $j_{1}, \ldots, j_{r}$, respectively (the case when two of these stable sets have the same centre can be easily reduced to this case). Let $W$ be the set of subsets with size two of $\left\{j_{1}, \ldots, j_{r}\right\}$. Therefore, for all $m \in\{1, \ldots, r\}, I_{i}^{m}-W$ has size at most $2 q+3-(r-1)=2 q+4-r$ since each centre $j_{m}$ belongs to $r-1$ elements in $W$. Besides, each element of $W$ belongs to exactly one set $I_{i}^{m}$ for $m \in\{1, \ldots, r\}$, since $S_{i}$ induces a complete subgraph. Then, $\left|I_{i}^{1} \cup \ldots \cup I_{i}^{r}\right| \leq$ $\left(\sum_{m=1}^{r}\left|I_{i}^{m}-W\right|\right)+|W| \leq r(2 q+4-r)+\frac{r(r-1)}{2}$.

Next, each remaining stable set (if exist) $I_{i}^{r+1}, \ldots, I_{i}^{q+2}$ has no centre, then $\left|I_{i}^{d}\right| \leq 3$ for all $d \in\{r+1, \ldots, q+2\}$. Thus, $\left|I_{i}\right| \leq r(2 q+4-r)+\frac{r(r-1)}{2}+3(q+2-r)=-\frac{r^{2}}{2}+r\left(2 q+\frac{1}{2}\right)+3(q+2)$. Since the last expression is non decreasing for $r \in\{1, \ldots, q+2\}$, we have that 


$$
\left|I_{i}\right| \leq-\frac{(q+2)^{2}}{2}+(q+2)\left(2 q+\frac{1}{2}\right)+3(q+2)=(q+2)\left(\frac{3}{2} q+\frac{5}{2}\right) .
$$

Therefore, $\left|I_{i}\right| \leq(q+2)\left(\frac{3}{2} q+\frac{5}{2}\right)$ for every $i=1, \ldots, 2 q+3$. Since $|I|=\sum_{i=1}^{2 q+3}\left|I_{i}\right|$, it follows that $|I| \leq(2 q+3)(q+2)\left(\frac{3}{2} q+\frac{5}{2}\right)$ and thus, $\alpha(K(2 q+4,2) \square K(2 q+4,2)) \leq(2 q+3)(q+2)\left(\frac{3}{2} q+\frac{5}{2}\right)$.

Let $t<\frac{2 q^{2}+18 q+24}{3 q+5}$. Assume that exists a 2-tuple coloring of the graph $K(2 q+4,2) \square K(2 q+4,2))$ with $2 q+t$ colors. Therefore, there exists an homomorphism from $K(2 q+4,2) \square K(2 q+4,2)$ to $K(2 q+t, 2)$.

Now, from the well known no-homomorphism Lemma 3, we have that,

$$
\alpha(K(2 q+4,2) \square K(2 q+4,2)) \geq \frac{\alpha(K(2 q+t, 2))|V(K(2 q+4,2) \square K(2 q+4,2))|}{|V(K(2 q+t, 2))|} .
$$

Then, $\alpha(K(2 q+4,2) \square K(2 q+4,2)) \geq \frac{(2 q+t-1) \cdot(2 q+4)^{2}(2 q+3)^{2} 2}{4(2 q+t)(2 q+t-1)}=\frac{(2 q+4)^{2}(2 q+3)^{2}}{2(2 q+t)}$.

Let us see that $\frac{(2 q+4)^{2}(2 q+3)^{2}}{2(2 q+t)}$ is greater than $(2 q+3)(q+2)\left(\frac{3}{2} q+\frac{5}{2}\right)$, which is a contradiction.

To this end, observe that if $t<\frac{2 q^{2}+18 q+24}{3 q+5}$, then

$$
\begin{gathered}
(2 q+3)(q+2)\left(\frac{3}{2} q+\frac{5}{2}\right)(2 q+t)=(2 q+3)(q+2)\left(\left(3 q^{2}+5 q\right)+\left(\frac{3}{2} q+\frac{5}{2}\right) t\right)< \\
\left.(2 q+3)(q+2)\left(\left(3 q^{2}+5 q\right)+\left(q^{2}+9 q+12\right)\right)=(2 q+3)(q+2)\left(4 q^{2}+14 q+12\right)\right)=\frac{(2 q+4)^{2}(2 q+3)^{2}}{2} .
\end{gathered}
$$

Therefore, $\chi_{2}(K(2 q+4,2) \square K(2 q+4,2)) \geq 2 q+t+1$.

Claim 1. Let $q>0$ be an integer. Then, $\frac{2 q^{2}+18 q+24}{3 q+5}$ is not an integer.

Proof. By polynomial division, we have that $2 q^{2}+18 q+24=(3 q+5)\left(\frac{2}{3} q+\frac{44}{9}\right)-\frac{4}{9}$. If $\left(2 q^{2}+18 q+24\right) /(3 q+5)$ is an integer, then $k=\left(\frac{2}{3} q+\frac{44}{9}\right)-\frac{4}{9(3 q+5)}$ is also an integer. Multiplying by 9 both terms in the last equality we have, $6 q+44=9 k+\frac{4}{3 q+5}$. As $q>0$ then, $0<\frac{4}{3 q+5}<1$ contradicting the assumption that $k$ is an integer.

Finally, since $\frac{2}{3} q+4<\frac{2 q^{2}+18 q+24}{3 q+5}$ and, by previous Claim $1, \frac{2 q^{2}+18 q+24}{3 q+5}$ is not an integer, then $\left\lceil\frac{2}{3} q\right\rceil+4<\frac{2 q^{2}+18 q+24}{3 q+5}$ and thus, we have finally that,

$$
2 q+\left\lceil\frac{2}{3} q\right\rceil+5 \leq \chi_{2}(K(2 q+4,2)) .
$$

As a corollary of Lemma 11 and by Corollary 1, we obtain the following result.

Corollary 4. $\chi_{2}(K(6,2) \square K(6,2))=8$.

Theorem 1. Let $k>n$ and let $t=\chi_{k}(K(m, n) \square K(m, n))$, where $m>2 n$. Then, either $t>m+2(k-n)$ or $t<m+(k-n)$. 
Proof. Suppose that $m+(k-n) \leq t \leq m+2(k-n)$. Therefore, there exists an homomorphism $K(m, n) \square K(m, n) \rightarrow K(t, k)$. Now, Stahl [11] showed that there is an homomorphism $K(m, n) \rightarrow K(m-2, n-1)$ whenever $n>1$ and $m \geq 2 n$. Moreover, it's easy to see that there is an homomorphism $K(m, n) \rightarrow K(m-1, n-1)$. By applying the former homomorphism $t-(m+(k-n))$ times to the graph $K(t, k)$ we obtain an homomorphism $K(t, k) \rightarrow K(2(m+k-n)-t, 2 k+m-n-t)$. Finally, by applying $2 k+m-t-2 n$ times the latter homomorphism to the graph $K(2(m+k-n)-t, 2 k+m-n-t)$ we obtain an homomorphism $K(2(m+k-n)-t, 2 k+m-n-t) \rightarrow K(m, n)$. Therefore, by homomorphism composition, $K(m, n) \square K(m, n) \rightarrow K(m, n)$ which contradicts Lemma 5 .

We can also obtain a lower bound for the $k$-tuple chromatic number of the graph $K(m, n) \square K(m, n)$ in terms of the clique number of $K(m, n)$. In fact, notice that $\omega(K(m, n) \square K(m, n))=\omega(K(m, n))=\left\lfloor\frac{m}{n}\right\rfloor$. Thus, we have the following result.

Theorem 2. Let $k>n$. Then, $\chi_{k}(K(m, n) \square K(m, n)) \geq k \omega(K(m, n))=k\left\lfloor\frac{m}{n}\right\rfloor$. In particular, if $n$ divides $m$ then, $\chi_{k}(K(m, n) \square K(m, n)) \geq m+(k-n) \frac{m}{n}$.

\section{Cases where $\chi_{k}(G \square H)=\max \left\{\chi_{k}(G), \chi_{k}(H)\right\}$}

Theorem 3. Let $G$ and $H$ be graphs such that $\chi(G) \leq \chi(H)=\omega(H)$. Then, $\chi_{k}(G \square H)=$ $\max \left\{\chi_{k}(G), \chi_{k}(H)\right\}$.

Proof. Let $t=\omega(H)$ and let $\left\{h_{1}, \ldots, h_{t}\right\}$ be the vertex set of a maximum clique $K_{t}$ in $H$ with size $t$. Clearly, $\chi_{k}(G) \leq \chi_{k}(H)=\chi_{k}\left(K_{t}\right)$. Let $\rho$ be a $k$-tuple coloring of $H$ with $\chi_{k}(H)$ colors. By equality (1), there exists a $t$-coloring $f$ of $G \square H$. Therefore, the assignment of the $k$-set $\rho\left(h_{f((a, b))}\right)$ to each vertex $(a, b)$ in $G \square H$ defines a $k$-tuple coloring of $G \square H$ with $\chi_{k}\left(K_{t}\right)$ colors.

Notice that if $G$ and $H$ are both bipartite, then $\chi_{k}(G \square H)=\chi_{k}(G)=\chi_{k}(H)$. In the case when $G$ is not a bipartite graph, we have the following results.

An automorphism $\sigma$ of a graph $G$ is called a shift of $G$ if $\{u, \sigma(u)\} \in E(G)$ for each $u \in V(G)$ [8]. In other words, a shift of $G$ maps every vertex to one of its neighbors.

Theorem 4. Let $G$ be a non bipartite graph having a shift $\sigma \in A U T(G)$, and let $H$ be a bipartite graph. Then, $\chi_{k}(G \square H)=\max \left\{\chi_{k}(G), \chi_{k}(H)\right\}$.

Proof. Let $A \cup B$ be a bipartition of the vertex set of $H$. Let $f$ be a $k$-tuple coloring of $G$ with $\chi_{k}(G)$ colors. Clearly, $\chi_{k}(G) \geq \chi_{k}(H)$. We define a $k$-tuple coloring $\rho$ of $G \square H$ with $\chi_{k}(G)$ colors as follows: for any vertex $(u, v)$ of $G \square H$ with $u \in G$ and $v \in H$, define $\rho((u, v))=f(u)$ if $v \in A$, and $\rho((u, v))=f(\sigma(u))$ if $v \in B$.

We may also deduce the following direct result.

Theorem 5. Let $G$ be an hom-idempotent graph an let $H$ be a subgraph of $G$. Thus, $\chi_{k}(G \square H)=\max \left\{\chi_{k}(G), \chi_{k}(H)\right\}=\chi_{k}(G)$. 
Let $A$ be a group and $S$ a subset of $A$ that is closed under inverses and does not contain the identity. The Cayley graph $\operatorname{Cay}(A, S)$ is the graph whose vertex set is $A$, two vertices $u, v$ being joined by an edge if $u^{-1} v \in S$. If $a^{-1} S a=S$ for all $a \in A$, then $\operatorname{Cay}(A, S)$ is called a normal Cayley graph.

Lemma 12 ([6]). Any normal Cayley graph is hom-idempotent.

Note that all Cayley graphs on abelian groups are normal, and thus hom-idempotents. In particular, the circulant graphs are Cayley graphs on cyclic groups (i.e., cycles, powers of cycles, complements of powers of cycles, complete graphs, etc). By Theorem 5 and Lemma 12 we have the following result.

Theorem 6. Let $\operatorname{Cay}(A, S)$ be a normal Cayley graph and let $C a y\left(A^{\prime}, S^{\prime}\right)$ be a subgraph of $\operatorname{Cay}(A, S)$, with $A^{\prime} \subseteq A$ and $S^{\prime} \subseteq S$. Then, $\chi_{k}\left(\operatorname{Cay}(A, S) \square \operatorname{Cay}\left(A^{\prime}, S^{\prime}\right)\right)=$ $\max \left\{\chi_{k}(\operatorname{Cay}(A, S)), \chi_{k}\left(\operatorname{Cay}\left(A^{\prime}, S^{\prime}\right)\right)\right\}$.

Definition 1. Let $G$ be a graph with a shift $\sigma$. We define the order of $\sigma$ as the minimum integer $i$ such that $\sigma^{i}$ is equal to the identity permutation.

Theorem 7. Let $G$ be a graph with a shift $\sigma$ of minimum odd order $2 s+1$ and let $C_{2 t+1}$ be a cycle graph, where $t \geq s$. Then, $\chi_{k}\left(G \square C_{2 t+1}\right)=\max \left\{\chi_{k}(G), \chi_{k}\left(C_{2 t+1}\right)\right\}$.

Proof. Let $\{0, \ldots, 2 t\}$ be the vertex set of $C_{2 t+1}$, where for $0 \leq i \leq 2 t,\{i, i+1 \bmod n\} \in$ $E\left(C_{2 t+1}\right)$. Let $G_{i}$ be the $i^{\text {th }}$ copy of $G$ in $G \square C_{2 t+1}$, that is, for each $0 \leq i \leq 2 t, G_{i}=\{(g, i)$ : $g \in G\}$. Let $f$ be a $k$-tuple coloring of $G$ with $\chi_{k}(G)$ colors. We define a $k$-tuple coloring of $G \square C_{2 t+1}$ with $\chi_{k}(G)$ colors as follows: let $\sigma^{0}$ denotes the identity permutation of the vertices in $G$. Now, for $0 \leq i \leq 2 s$, assign to each vertex $(u, i) \in G_{i}$ the $k$-tuple $f\left(\sigma^{i}(u)\right)$. For $2 s+1 \leq j \leq 2 t$, assign to each vertex $(u, j) \in G_{j}$ the $k$-tuple $f(u)$ if $j$ is odd, otherwise, assign to $(u, j)$ the $k$-tuple $f\left(\sigma^{1}(u)\right)$. It's not difficult to see that this is in fact a proper $k$-tuple coloring of $G \square C_{2 t+1}$.

\section{References}

[1] M. O. Albertson, K. L. Collins. Homomorphisms of 3-chromatic graphs. Discrete Mathematics, 54:127-132, 1985.

[2] C. Berge. Graphs and Hypergraphs. North-Holland, Amsterdam, 1976.

[3] B. Bollobás, A. Thomason. Set colourings of graphs. Discrete Mathematics, 25(1):21-26, 1979.

[4] P. Erdős, C. Ko, R. Rado. Intersection theorems for systems of finite sets. Quarterly Journal of Mathematics, 12:313-320, 1961.

[5] C. D. Godsil, G. Royle. Algebraic graph theory. Graduate Texts in Mathematics. Springer, 2001. 
[6] G. Hahn, P. Hell, S. Poljak. On the ultimate independence ratio of a graph. European Journal on Combinatorics, 16:253-261, 1995.

[7] A. J. W. Hilton, E. C. Milner. Some intersections theorems for systems of finite sets. Quarterly Journal of Mathematics, 18:369-384, 1967.

[8] B. Larose, F. Laviolette, C. Tardif. On normal Cayley graphs and Hom-idempotent graphs, European Journal of Combinatorics, 19:867-881, 1998.

[9] L. Lovász. Kneser's conjecture, chromatic number and homotopy, Journal of Combinatorial Theory, Series A, 25:319-324, 1978.

[10] G. Sabidussi. Graphs with given group and given graph-theoretical properties. Canadian Journal of Mathematics, 9:515-525, 1957.

[11] S. Stahl. n-Tuple colorings and associated graphs. Journal of Combinatorial Theory, Series B, 20:185-203, 1976.

[12] S. Stahl. The multichromatic numbers of some Kneser graphs. Discrete Mathematics, 185:287-291, 1998. 